\title{
A narrative review for the Hippo-YAP pathway in cancer survival and immunity: the Yin-Yang dynamics
}

\author{
Ruochong Wang, Guiquan Zhu^ \\ Department of Head and Neck Oncology, West China Hospital of Stomatology, Sichuan University, Chengdu, China \\ Contributions: (I) Conception and design: R Wang; (II) Administrative support: G Zhu; (III) Provision of study materials or patients: None; (IV) \\ Collection and assembly of data: R Wang; (V) Data analysis and interpretation: R Wang; (VI) Manuscript writing: Both authors; (VII) Final approval \\ of manuscript: Both authors. \\ Correspondence to: Guiquan Zhu. Department of Head and Neck Oncology, West China Hospital of Stomatology, Sichuan University, No. 14, Section \\ 3, Renmin South Road, Chengdu 610041, China. Email: zhugq@scu.edu.cn.
}

\begin{abstract}
The Hippo-YAP pathway is fast becoming a key instrument in regulating cancers. Binary oppositions, also known as the Yin-Yang dynamics in Chinese, have emerged in its effects. Some oppositions are attributable to in vitro and in vivo experimental conditions, some are due to differences between cancers or species, some are derived from its inherent duality endowed by upstream and downstream signaling, and some are yet unresolved mysteries. However, as bewildering they are, few have been noticed and defined so far. In this review, we first look back on the Hippo pathway which was initially identified more than a decade ago. We then focus on tumor biology, especially some latest popular mechanisms that regulate tumor cell survival, such as ferroptosis, autophagy, and apoptosis. The third chapter is concerned with the findings of the relationship between the Hippo pathway and tumor immunity on the microenvironment in which tumor cells progress. In each of these main sections the contradictory points of the Hippo pathway are elucidated and thoroughly examined. On one hand, the Yin-Yang dynamics of the Hippo pathway brings about considerable challenges for current research; on the other hand, this work will generate fresh insight into it and offer opportunities for subsequent drug development for cancer and regenerative medicine.
\end{abstract}

Keywords: Hippo pathway; cancer; ferroptosis; autophagy; apoptosis

Submitted Sep 05, 2021. Accepted for publication Dec 02, 2021.

doi: $10.21037 /$ tcr-21-1843

View this article at: https://dx.doi.org/10.21037/tcr-21-1843

\section{Introduction}

The Hippo pathway was first described in Drosophila melanogaster as a tumor suppressor $(1,2)$, which could inhibit the cell growth and normalize the organ size (3). Later, it was found that the Hippo pathway was wellconserved in mammals and played the same role as in Drosophila melanogaster, e.g., managing cell behavior, dictating cell differentiation, and maintaining tissue homeostasis. More profoundly, the Hippo pathway perturbation and mutation have been observed in many types of human cancers, including lung cancer, colorectal cancer, breast cancer, and liver cancer, etc. $(4,5)$.

But it is still debatable whether the Hippo pathway gives rise to cancer or acts as a tumor suppressor. The specific mechanisms that regulate the contradicting activity of the pathway are under intensive investigation. In this paper, we will give a brief overview on molecules of the Hippo pathway and summarize the current knowledge on the two faces of the Hippo-YAP pathway on cancer progression. We present the following article in accordance with the Narrative Review reporting checklist (available at https://

\footnotetext{
$\wedge$ ORCID: 0000-0002-8316-5809.
} 


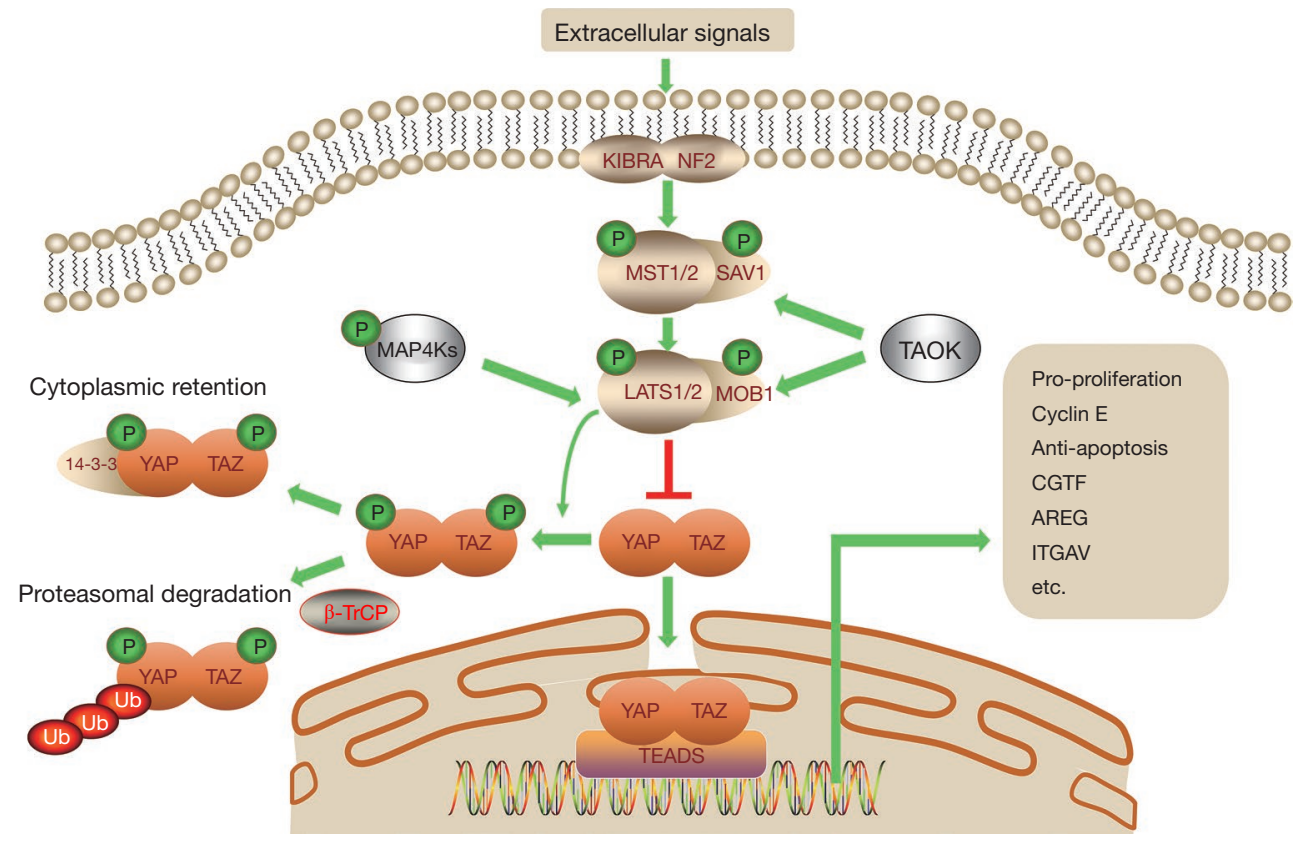

Figure 1 The Hippo-YAP pathway in mammals. Various extracellular signals regulate YAP/TAZ phosphorylation and nuclear localization via the recruitment of MST1/2 (large tumor suppressor 1/2)-LATS1/2 (mammalian STE20-like protein kinase 1/2) kinase cascade by KIBRA and neurofibromatosis type 2 (NF2). Other proteins, such as the adaptor proteins Salvador homologue 1 (SAV1), MOB kinase activator 1 (MOB1), the mitogen-activated protein kinase kinase kinase kinase (MAP4K) and TAO kinases (TAOK) take parts in the cascade. If signals activate the core of the Hippo pathway, it brings about the cytoplasmic retention and proteasomal degradation of YAP/TAZ, but if they inhibit the core, YAP/TAZ enter the nucleus, bind to the corresponding DNA-binding protein, which is most likely TEA domain (TEAD) proteins, and promote the target gene transcription, including connective tissue growth factor (CTGF), integrin- $\alpha \mathrm{V}$ (ITGAV), amphiregulin (AREG).

tcr.amegroups.com/article/view/10.21037/tcr-21-1843/rc).

\section{The canonical Hippo-YAP pathway members}

The Hippo pathway is composed of a large network of proteins with serine/threonine kinase activity (5-7), including neurofibromatosis type 2 (NF2) (homologs of Drosophila Merlin), mammalian STE20-like protein kinase 1 (MST1. also known as STK4), and MST2 (also known as STK3) (homologs of Drosophila Hippo), large tumor suppressor 1 (LATS1), LATS2 (homologs of Drosophila Warts), the adaptor proteins Salvador homologue 1 (SAV1), MOB kinase activator 1A (MOB1A), and MOB1B (Figure 1). Recruitment of NF2 to the plasma membrane initiates the phosphorylation of MST and LATS, which leads to phosphorylation of YAP and TAZ $(8,9)$.

MST1/2 exist in a complex with SAV1, which phosphorylates and activates the downstream kinase MOB/ LATS1/2 complex. In parallel to MST1/2, the mitogen- activated protein kinase kinase kinase kinase (MAP4K) (homologs of Drosophila Hppy) family and TAO kinases (TAOK) perform the same function (10). The activated LATS1/2 phosphorylates YAP/TAZ on five (YAP) and four (TAZ) conserved serine residues (6), leading to its cytoplasmic retention by $14-3-3$ and $\beta$-TrCP-mediated ubiquitination and proteasomal degradation.

On the other hand, when the Hippo pathway kinase cascade is inactivated, YAP and TAZ are dephosphorylated and translocate into the nucleus where $\mathrm{YAP} / \mathrm{TAZ}$ regulate genes associated with cell growth, proliferation, survival, and metabolism. Without DNA-binding domain, YAP/TAZ play their roles by interacting with transcription factors, such as ErbB4, Runx2, Smad, TEAD, and p73. The major partner of YAP/TAZ is the TEA domain (TEAD) proteins (homologs of Drosophila Sd). Target genes of YAP and TEAD include connective tissue growth factor $(C T G F)(11)$, integrin- $\alpha \mathrm{V}(I T G A V)(12)$, amphiregulin $(A R E G)(13,14)$, etc. In the absence of nuclear YAP/TAZ, TEADs bind to 


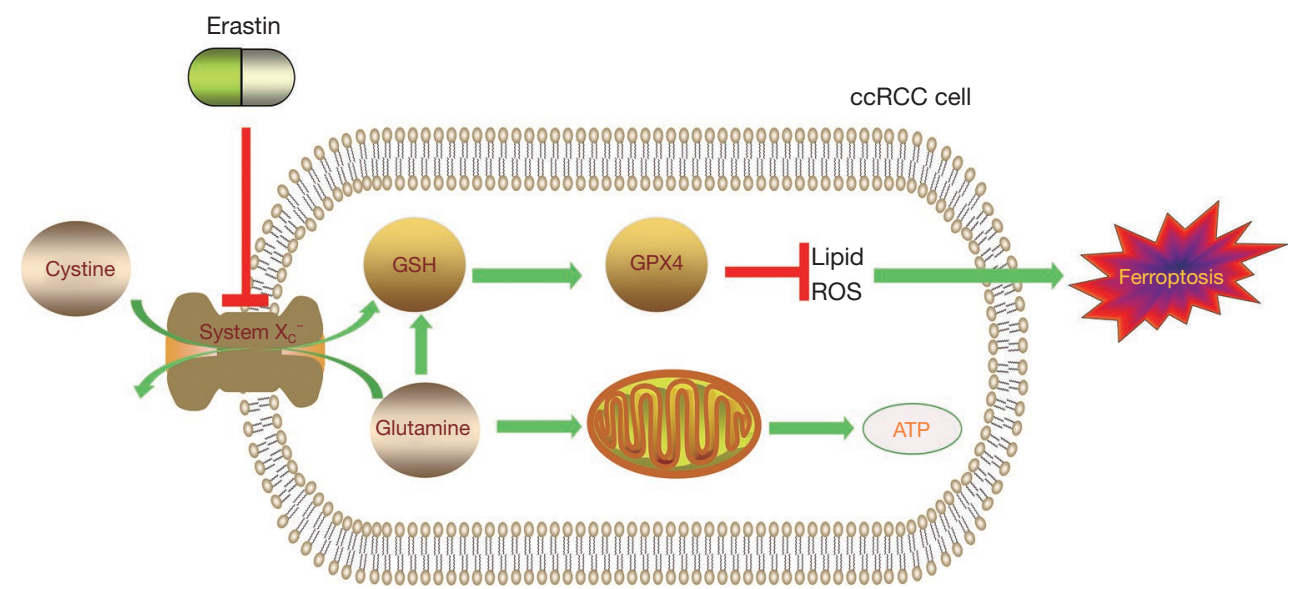

Figure 2 Clear cell renal cell carcinoma (ccRCC) cells are highly sensitive to the depletion of cystine and glutamine. Erastin inhibits system $\mathrm{X}_{\mathrm{C}}^{-}$, thus blocking the import of cystine, causing glutathione (GSH) depletion and glutathione peroxidase 4 (GPX4) inactivation. Uncontrolled lipid peroxidation generates reactive oxygen species (ROS), ultimately resulting in ferroptosis. Furthermore, recent studies have found that ccRCC cells rely mainly on glutamine for adenosine triphosphate (ATP) generation. Ferroptosis becomes more severe as ccRCC cells use up glutamine.

$\mathrm{Vg}$ domain-containing transcriptional cofactor-like protein 4 (VGLL4) (homologs of Drosophila Tgi), and thereby inhibit the expression of these target genes (15).

\section{Hippo-YAP-regulated tumor survival}

\section{Ferroptosis}

Ferroptosis is a newly discovered and utterly distinctive death form, first observed when RAS-selective lethal small molecule erastin brought about it in cancer cells (16). When later digging into the mechanisms of it, researchers found the eradication process was featured with the accumulation of ROS and mere iron-dependency. Ferroptosis was thus given the name (17). Glutathione peroxidase 4 (GPX4) (18) is a key element in this process. GPX4 is a membrane lipid repair enzyme under normal circumstances, capable of reducing lipid peroxides in a complex cellular membrane environment (19). When it malfunctions, it fails to limit the growth of lipid-based reactive oxygen species (ROS), incurring ferroptosis (20,21). Erastin inactivates GPX4 through glutathione (GSH) depletion (18). Specifically, erastin inhibits system $X_{C}^{-}$, a 1:1 cystine:glutamate exchange antiporter that blocks cystine uptake and reduces glutamate efflux at the same time (22). System $\mathrm{X}_{\mathrm{C}}{ }^{-}$can thus control GSH synthesis by regulating glutamine and cystine. In clear cell renal cell carcinoma (ccRCC), cancer cells have been identified highly sensitive to the depletion of glutamine and cystine (23). Furthermore, when taking cystine aside, glutamine can still do the solo dance in the death process. It was found that ablating glutaminolysis can protect heart tissue from ischemia-reperfusion injury caused by ferroptosis (24) (Figure 2).

Another in vitro study (25) found that erastin triggered severer cell death in $3 \mathrm{D}$ tumor spheroids formed by MDA231 and H1650 cells as the cell density increased, leading to the conclusion that high cell density more rapidly depletes glutamine. Li et al. (26) summarized that glutamine metabolic pathways play a regulatory role in ferroptosis.

What makes it more intriguing is that some cancer cells show higher uptake of glutamine than T cells and myeloid cells do (27). This phenomenon contradicts the Warburg effect, which is widely considered significant in tumor cells for adenosine triphosphate (ATP) generation through glycolysis (28). The way they applied ccRCC for measuring the consumption of glucose and glutamine resembles that of Miess et al. (23), who demonstrated that renal cancer cells are highly dependent on the GSH/GPX pathway to stay alive. Therefore, for RCC, glutamine is essential for tumor metabolism. Otherwise, the deficiency of glutamine would not only lead to impaired ATP production but also make cancer cells susceptible to ferroptosis.

Scientists agree that YAP/TAZ increase sensitivity to ferroptosis and that Hippo signaling pathway suppresses ferroptosis. The molecular mechanism behind this relationship has been hunted down to several ferroptosis 


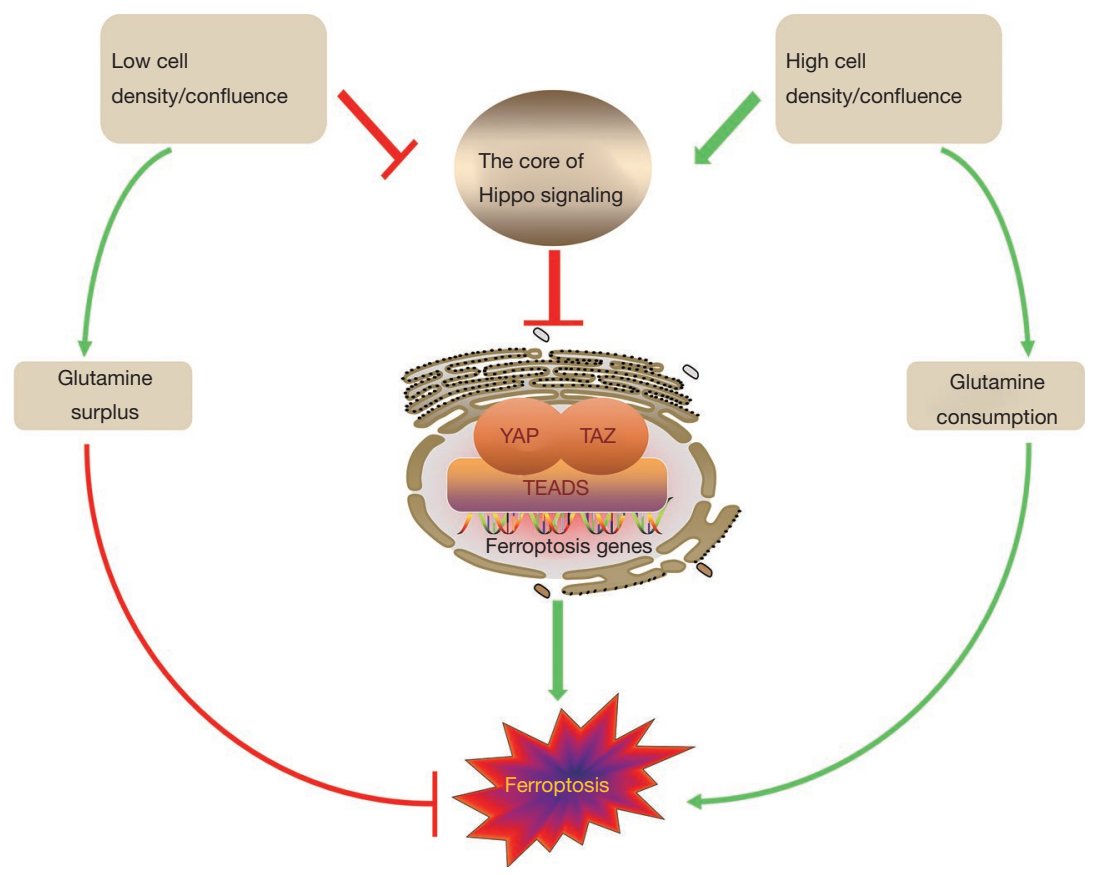

Figure 3 'Ferroptosis on' in low-density/confluence cells, and 'ferroptosis off' in high-density/confluence cells. As the number of cancer cells increases, more glutamine will be consumed. However, instead of being more sensitive to ferroptosis, cancer cells develop resistance to ferroptosis. This is due to the inhibition of YAP activation in high-density/confluence cells and reduced expression of ferroptosis genes, such as ACSL4 and TFRC. When there are fewer tumor cells, their metabolism scarcely requires excessive consumption of glutamine. YAP is activated to promote cell proliferation, but, as inferred from the scenario of high cell density/confluence, it also promotes ferroptosis.

modulators, such as ACSL4, TFRC $(25,29)$ and SLC7A11 (30).

Despite these solid findings, the actual anti-tumor effect of YAP-promoting ferroptosis is still debating. Wu et al. (25) demonstrated that except for MDA231 cells, the cell death rate went down as the cell number rose up, because the expression of ACSL4 and TFRC decreased along with the increase of cell density. Yang et al. (31) found that the sensitivity to ferroptosis was influenced by cell density as well, and that low-density but not high-density ovarian cancer cells were highly sensitive to ferroptosis. Similarly, with the confluence of HCT116 cells increasing, increased phosphorylation and decreased nuclear localization of YAP rendered cancer cells resistant to ferroptosis. It is because E-cadherin (ECAD) is required for building intercellular contact in cells (32) and that ECAD is able to activate the intracellular NF2 and Hippo signaling pathway. With the activation of NF2 and Hippo signaling, YAP/TAZ entry into the nucleus is inhibited, preventing the target genes, ACSL4 and TFRC, from playing a role in promoting ferroptosis. Though glutamine deprivation is the trigger for ferroptosis, depleting glutamine yet fails to restore ferroptosis in these confluent cells. The reason is that highdensity or high-confluence cells in the tumor setting do not die from ferroptosis while consuming glutamine (Figure 3). Thus, other than YAP-activated tumors, tumors irrelevant to the Hippo pathway perturbation are virtually unaffected by ferroptosis.

When YAP is needed for exerting its repair and regenerative functions at low cell density or confluence, cells are instead sensitive to ferroptosis. Of course we cannot guarantee this since the biological function of ferroptosis still remains unexplained under normal physiological conditions (30).

Except for that new perspective on glutamine metabolism, most past and recent studies have been based on the Warburg effect. Significant implications as the new view may have in imaging, the veracity of previous experiments should not be ignored. Thus, we elucidate the related mechanism from the view of glucose metabolism. First, aerobic glycolysis increases global O-linked beta$\mathrm{N}$-acetylglucosaminylation (O-GlcNAcylation). As a monosaccharide-based modification, Uridine-diphosphate 
$\mathrm{N}$-acetylglucosamine (UDP-GlcNAc) is its only sugar donor, which is synthesized from glucose through the hexosamine biosynthetic pathway (HBP) (33). It has been identified that O-GlcNAcylation at the Ser109 site (34) and Thr241 site (35) of YAP stabilized YAP by antagonizing its Hippo-dependent phosphorylation, which incurred highglucose-induced liver tumorigenesis. Interaction between YAP and O-GlcNAcylation links glucose abundance to Hippo signaling activity and tumorigenesis (36). Besides, YAP upregulates enzymes in glycolysis (37), including some critical rate-limiting enzymes. A positive loop therefore forms between YAP and glycolysis. Accordingly, as tumor cells consumed glucose, ferroptosis was eventually enhanced as well, proven by an increase in TFRC expression elicited by YAP-O-GlcNAcylation (30).

Surprisingly, erastin, Sorafenib and other system $\mathrm{X}_{\mathrm{C}}{ }^{-}$ inhibitors could downregulate YAP at both the mRNA level and the protein level (38). This finding seems contradictory compared to the literature of $\mathrm{Wu}$ et al. (25). The authors (38) made a clarification that two theories were complementary to each other because the TEAD-based transcription program stimulated by YAP was a prerequisite for triggering ferroptosis, while the degree of ferroptosis was determined by the remaining YAP levels. They used in vitro study to prove that it was the accumulated endogenous glutamate in the lung adenocarcinoma (LUAD) cell that triggered the suppression of YAP through ADCY10/PKA/GFPT1 signaling axis, in which GFPT1 was the rate-limiting enzyme of HBP. Meanwhile, the data about xenografts generated by H1975 and A549 cells validated that the suppression of YAP induced the inhibition of ferroptosisassociated tumor growth.

Therefore, in the entire process of ferroptosis, YAP is critical for regulating both the initiation and progression of it. Firstly, YAP exerts a tumor-suppressive effect, but later it exerts an oncogenic effect.

\section{Autophagy}

Autophagy is not only a form of cell death, but also a mechanism of cell survival under stress, e.g., hypoxia, nutrient limitation, and redox stress $(39,40)$. In normal cells and tissues, autophagy resembles quality inspection system, which maintains cellular homeostasis through wiping out damaged organelles and proteins. The process is regulated by more than 30 autophagy-related genes (ATG) (41). After catabolism, degradation products are recycled to the cytoplasm for cellular replenishment. In an in vivo study, conditional (acute) systemic (whole-body) Atg7 deficiency in adult mice resulted in a depletion of dedicated nutrient stores during fasting. Without exogenous nutritional supplementation, this systematic metabolic defect may cause lethal hypoglycemia (42).

Autophagy has the potential to fuel almost all aspects of central carbon metabolism through mediating the degradation of various macromolecular complexes, such as carbohydrates, proteins, DNAs, and lipids. The main catabolic process is carried out by the degradative enzymes from lysosomes in autolysosomes that are formed by the fusion of autophagosomes and lysosomes (43-45).

Even though autophagy has been extensively studied, effects of autophagy on tumor development remain contradictory. For example, there are two conflicting papers published in Nature. One (46) declared that depletion of Atg5/7 impaired metabolism, proliferation, survival and malignancy of spontaneous tumors in autologous cancer models; but the other one (47) introduced the concept of replication crisis and noted that autophagy, if inhibited, could lead to tumors bypassing the replication crisis, which is necessary for the initiation of cancer. Wen et al. (48) confessed that they still did not know whether autophagy promoted or inhibited cancer. Kimmelman et al. (49) also reviewed that autophagy was initially thought to have a tumor-suppressive role, capable of removing damaged or redundant organelles and eliminating ROS. More recently, however, autophagy has been found to have a key tumorigenic role, particularly in promoting tumor metabolism in a variety of cancer types. It has been demonstrated that autophagy could help tumors to cope with low-nutrient conditions and chemotherapy-induced stress (50).

Leaving aside the dual role of autophagy on tumors, the relationship between the Hippo-YAP pathway and autophagy is quite contradictory as well. High mobility group box 1 (HMGB1), a renowned regulator of autophagy (51), can be enhanced by YAP, resulting in glioma progression. In a simulated tumor microenvironment (TME) with high cell density and high cell confluence, YAP/TAZ double knockdown inhibits autophagosome formation, and autophagy promotes the cell proliferation with activated YAP/TAZ.

Conversely, in liver cancer development, impaired autophagy triggers YAP stabilization, while YAP can be degraded by autophagy (52). In colorectal cancer (CRC) (53), YAP-TEAD complex upregulates the transcription of the apoptosis-inhibitory protein Bcl-2, 
which subsequently shuns autophagy-related cell death and promotes CRC progression. Furthermore, rapamycinactivated autophagy counteracts YAP to exert its function.

Pavel et al. (54) summarized that autophagy compromise could either activate or inhibit YAP/TAZ in different cell lines. Downregulation of YAP/TAZ upon autophagy suppression was observed in MCF10A, HEK293T, HeLa, and $\mathrm{pMECs}$ cells, while upregulation of YAP/TAZ activity was spotted in HepG2 and THLE2 cells. Then it was found that CTNNA1 determined the function of YAP/TAZautophagy axis. In cells where autophagy and YAP/TAZ were cooperative, the relative ratio of CTNNA1 basal levels was higher than 0.5 , while in cells where autophagy was antagonistic to YAP/TAZ, it was around 0.3 or less. Thus CTNNA1, as one of $\alpha$-catenins, determines the differential effects of autophagy on YAP/TAZ activity.

\section{Apoptosis}

Compared to autophagy and ferroptosis, apoptosis is now a less popular or controversial topic when it comes to the relationship with cancers. A fair amount of literature has suggested that apoptosis and the Hippo-YAP pathway were closely linked. By inducing cell-cycle arrest, cellular senescence and apoptosis, the p53 family proteins take a tremendous part in tumor suppression, among which the role of p53 is particularly prominent (55). Other family members include p63 and p73, two homologs of p53 that share homology in DNA sequence, protein structure and functional features (56).

Apoptosis is a vital mechanism to eliminate oncogenesis. p53 earns its title 'Guardian of the genome' (57) because of its ability to detect DNA damage, hypoxia, and oncogenic events, and to eliminate them through cell-cycle arrest, senescence, apoptosis $(58,59)$. In fact, over half of human cancers harbors mutant p 53 (60).

In an earlier report (61), LATS2 activated p53 in the nucleus and transcription of LATS2 was positively regulated by $\mathrm{p} 53$. Then, Aylon et al. (62) came up with an indirect and novel way LATS2 regulated the p53 pathway. Apoptosisstimulating protein of p53-1 (ASPP1) plays a key role in proapoptotic response. LATS2 phosphorylates ASPP1 and drives its nuclear localization in HCT116 cells, shunting p53 to the proapoptotic promoter. YAP antagonizes the tumor suppression function of the LATS2/ASPP1/p53 axis by disrupting LATS2-ASPP1 binding. Vigneron et al. (63) confirmed it from the opposite side: YAP would remain nuclear and ASPP1 would remain cytoplasmic in cancers with reduced LATS2 activity. Additionally, LATS1/2 can bind to murine double minute2 (MDM2) and inhibit it, which is the first identified inhibitor of $\mathrm{p} 53$; hence the stabilization of p53 $(61,64,65)$ (Figure $4 A)$. It seems that LATS1/2 does suppress oncogenesis by promoting $\mathrm{p} 53$, while YAP stands against this anti-tumor process.

p63 consists of full-length transactivation domain (TA) isoform, TAp63, and the amino-deleted $\Delta \mathrm{N}$ isoform. The paradox is that the former structurally and functionally resembles $\mathrm{p} 53$, while its counterpart, $\Delta \mathrm{Np} 63$, facilitates oncogenesis by indirectly affecting YAP nuclear transposition in head and neck squamous carcinoma $(66,67)$, as evidenced by the overexpression of $\triangle \mathrm{Np} 63$ and YAP in this kind of cancer (68).

In response to genotoxic stresses, $\mathrm{p} 73$ functions similarly to p53 $(69,70)$. Firstly, the non-receptor tyrosine kinase c-Abl (ABL1), activated by stresses, phosphorylates p73 at $\mathrm{Tyr}^{99}$ and initiates the DNA damage-induced apoptosis $(71,72)$. There is also an indirect way to apoptosis. Activated c-Abl can phosphorylate YAP at $\mathrm{Tyr}^{357}$ (73). The modified YAP preferentially associates with $\mathrm{p} 73$ in targeting apoptotic gene promoters $(74,75)$ and stabilizes it by preventing nuclear export and subsequent degradation (76). It should be noted that the interaction of $\mathrm{p} 73$ with $\mathrm{Abl}$ and YAP is an independent event, as binding to c-Abl and tyrosine phosphorylation of $\mathrm{p} 73$ are not required for association with YAP (77) (Figure 4B). Nevertheless, such interaction differs from YAP in the p53 pathway: c-Ablmediated phosphorylation of YAP is proapoptotic (73), and it can be deducted that LATS1/2 is not proapoptotic in the p73 pathway, since LATS1/2 phosphorylates YAP at different sites. Despite the oncogenic and pro-proliferative property of YAP, YAP can be a tumor suppressor (77). Another research (78) also validated the idea. It proved that under DNA damage, the reintroduction of YAP in acute lymphoblastic leukemia (ALL) (Jurkat) or AML (OCI/AML3) reduced cell proliferation and induced apoptosis, while the overexpression of LATS prevented the phosphorylation of p73 and inhibited apoptosis. Still, in the absence of this condition, YAP prefers to interact with the oncogenic transcription modulator RUNX, bringing about increased degradation of $\mathrm{p} 73$ (79).

Apart from ABL1-centered apoptosis, RAS association domain family $1 \mathrm{~A}$ (RASSF1A) elicits apoptosis through the p73 pathway as well (80). A previous study (81) demonstrated that the Raf1 proto-oncogene bound to MST2, preventing its activation and proapoptotic signaling. RASSF1A can abrogate the Raf1-MST2 complex, and downregulation 

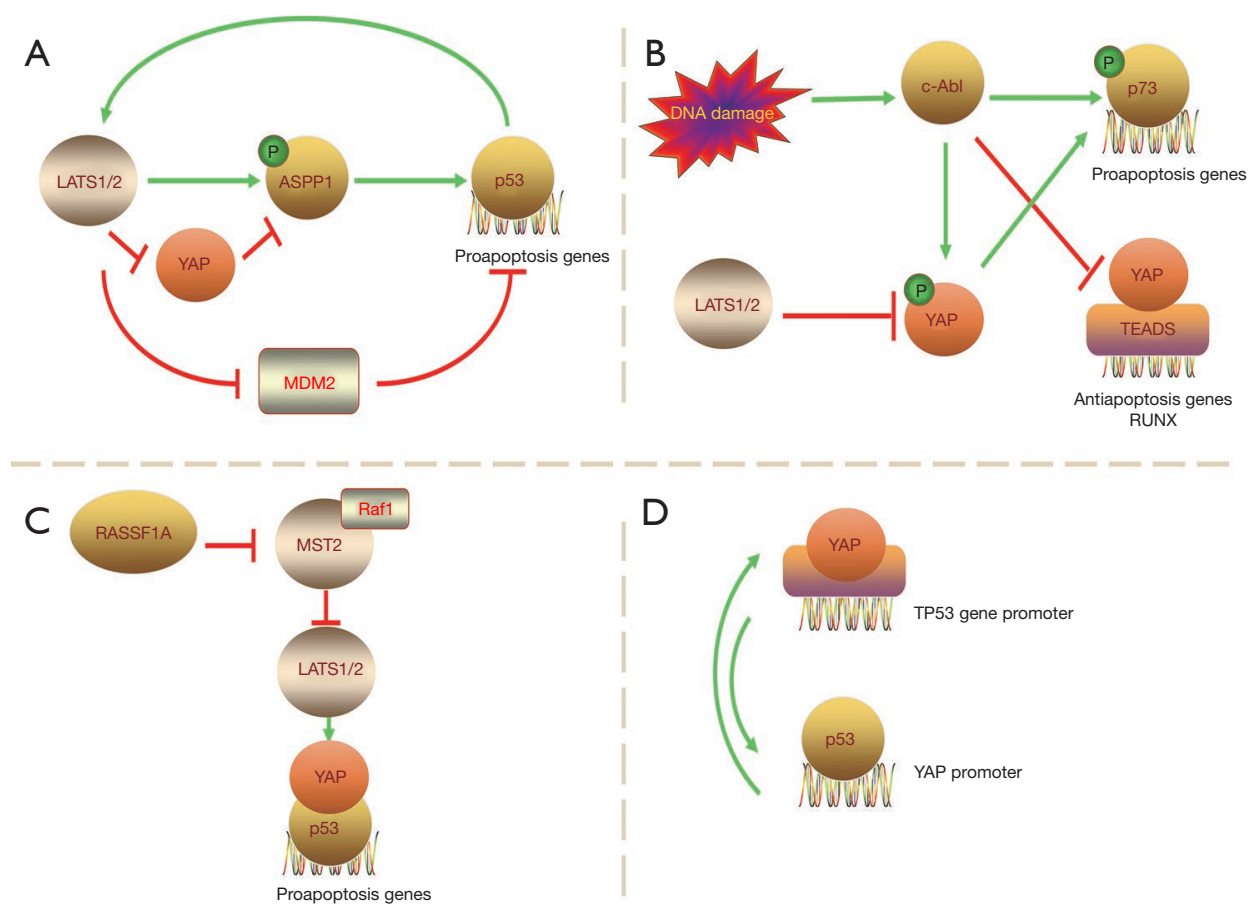

Figure 4 The conflicting relationship between the Hippo pathway and apoptosis. (A) LATS1/2 helps p53 bind to the proapoptotic promoter by promoting apoptosis-stimulating protein of p53-1 (ASPP) entry into the nucleus and inhibiting YAP and murine double minute2 (MDM2); at the same time, p53 also actively regulates LATS2, thus forming a positive loop of LATS1/2 and p53. (B) Having received the signal of genotoxic stress, the non-receptor tyrosine kinase c-Abl phosphorylates p73 and YAP at different tyrosine sites, different from the phosphorylation by LATS, the latter being able to stabilize p73 after phosphorylation. Furthermore, c-Abl is a negative regulator of TEADs, which co-activates anti-apoptotic target genes with YAP. (C) The MST2-Raf1 complex prevents MST2 activation. The complex can be abolished and the inactivation can be reversed by RAS association domain family 1A (RASSF1A). When RASSF1A is present, LATS1/2 does not isolate YAP outside the nucleus, but facilitates its entry into the nucleus and binding to p73, thereby transcribing proapoptotic genes. Although YAP is not phosphorylated at tyrosine sites at this point, it exerts a proapoptotic effect. It is LATS1/2 that plays the 'the Yin-Yang dynamics' role. (D) Instead of dampening apoptosis by disrupting LATS2-ASPP1 binding, YAP upregulates p53 expression, bringing about apoptosis by directly binding to TP53 gene promoter, but the transcription factor remains unknown. p53 can bind to the YAP promoter and upregulate YAP expression as well, forming another positive loop.

of endogenous MST2 in MCF7 decreases RASSF1Ainduced apoptosis (80) (Figure 4C). Thus, MST2 may serve as a hub that functionally connects proliferative and proapoptotic pathways. More interestingly, the authors (80) suggested that LATS1 also had a dual role. It is widely acknowledged that LATS1/2 results in the cytoplasmic sequestration and proteasomal degradation of YAP/TAZ, but in response to RASSF1A, it may help YAP bind to p73 and nuclear translocation (80).

There are some more dizzy contradictions. For example, unlike epithelial carcinomas, hematologic malignancies do not appear to require p53 inactivation, but they need the early inactivation of the ABL1-YAP-p73 axis.
However, in chronic myeloid leukemia (CML), ABL1 can be tumorigenic since it is also a pro-proliferative factor if its activation goes uncontrollable (82-84); For WT and p53-mutant MM (multiple myeloma) cell lines, MST1 knockdown recovers apoptosis, suggesting that restoring YAP levels could be a new treatment strategy in tumors with p53 inactivation (78); Likewise, a positive feedback loop also exists between YAP and p53: YAP can bind to TP53 gene promoter and upregulate $\mathrm{p} 53$ expression, resulting in apoptosis. In turn, p53 can bind to the YAP promoter and upregulate YAP expression (Figure 4D). This feedback loop serves as an important part to increase chemosensitivity of hepatocellular carcinoma cells $(58,85)$. 


\section{Cell proliferation}

Uncontrolled cell proliferation is often the first step in the development of cancer. The inhibition of cell proliferation by activation of the Hippo pathway was established as early as it was discovered. Under physiological conditions, the Hippo pathway is well established for size control of organs (3). This trait was initially applied in regenerative medicine to promote regeneration of damaged tissues and proliferation of stem cells by activating YAP. Specific mechanisms include inhibition of differentiation, expansion of the stem cell pool, as well as reprogramming differentiated cells into a more primitive and stem celllike progenitor cell state (86). It has been confirmed that in developing skin, ectopic YAP activation causes decreased expression of differentiation markers (Krt1, Ivl and Lor) and increased expression of progenitor cell markers (87). Another important effect is its involvement in facilitating the entry of non-dividing cells or quiescent cells into the cell cycle. Activation of YAP/TAZ has been applied to stem cell expansion in injured intestine, oval cell expansion, hepatocyte injury, and even regeneration of the cardiomyocyte after myocardial infarction $(88,89)$, which is one of the typical quiescent cells.

It was subsequently found that this function and cancer development are also closely related. When the Hippo pathway kinase is mutated and loses its inhibitory effect on YAP/TAZ, YAP/TAZ promote the growth of cells and the tumorigenic potential by binding to TEADs and mediating the downstream target genes, including growth promoter such as ANKRD1 (90), Fos (91), cell cycle regulators such as FOXM1 (92), p27 (93), and apoptosis regulator such as Diap1 (94), NR4A1 (95). Accordingly, YAP is frequently amplified or overactivated in some human solid tumors. The truth is the stem cell properties, or 'stemness', conferred by YAP, finally leads to tumorigenesis plus other pro-tumorigenic events (88).

The process of YAP activation leading to tissue overgrowth and tumor formation is reversible after cessation of YAP activation (9). Therefore, this finding provides a solution to balance the treatment of damaged tissues with the prevention of tumorigenesis. As noted by Moya et al. (88), there existed a therapeutic window of YAP/ $\mathrm{TAZ}$ activation that could be used to induce regeneration while avoiding organ overgrowth.

\section{Hippo-YAP-regulated tumor immunity}

The Hippo-YAP pathway is not only associated with cell proliferation, but also implicated in the suppression of host antitumor immune response, including both innate immunity and adaptive immunity. Rather through the canonical path, many molecules in the Hippo-YAP pathway regulate immune cell activity and function independently.

First, in terms of innate immunity, the Hippo-YAP pathway plays an important role in anti-tumor response through detecting tumor DNA, inducing IFN-1, proinflammatory cytokines and chemokines, recruiting inflammatory cells, and subsequently stimulating adaptive immune responses $(96,97)$. Genes related to the Hippo-YAP pathway include S1PR1, PDGFB, and NLRP3 (98); proinflammatory cytokines and their receptors include IL $1 \alpha / \beta$, IL8, CXCR4, CXCL1/2/3/5, CCL2, and CSF1 (98-100); complement factors include CFI, C3 (98), and cells include myeloid-derived suppressor cells (MDSCs) (101), tumorassociated macrophages (TAMs) and M2 macrophages (102).

The Hippo pathway functions in tumorigenesis through regulating the tumor immune microenvironment rather than intra-tumor cell signaling. On the one hand, the Hippo-YAP pathway can be activated by innate immune receptors. Some innate immune receptor ligands, such as poly (I:C), LPS and CpG, are efficient anti-tumor agents, which can induce strong activation of IFN signaling and activation of the Hippo-YAP pathway (103). On the other hand, Hippo-YAP pathway dysregulation can in turn lead to cancer development through YAP accumulation and inhibition of anti-tumor response. Wang et al. (101) found that in a murine prostate adenocarcinoma model, YAP directed MDSCs to tumor sites via the CXCL5-CXCR2 axis to help establish the TME. In a murine liver tumor model, Hagenbeek et al. (104) also identified that TAZ induced myeloid cell infiltration and pro-inflammatory cytokine secretion. Apart from that, YAP can either direct TAMs differentiation to an immunosuppressive/"protumor" M2 phenotype or directly recruit M2 macrophages to suppress immune clearance (105). In most cases, the Hippo signaling is a positive factor in the innate immune response, but with the establishment of the TME, YAP/ TAZ dismisses the role of the Hippo signaling in antitumor immune responses.

Second, in adaptive immunity, many clinical successes have dictated that anti-tumor immunity is T-cell centered (96). Rather than function in the TME, YAP/ TAZ directly regulate immune cells, of which the $T$ cell is the main victim. Disruption of MST1/2 significantly reduces the level of peripheral $\mathrm{T}$ cells and impairs the function of T cells $(97,106)$. Overexpression of YAP induces 
differentiation of CD4+ T lymphocytes to Regulatory T cells (Treg) (107) and also negatively regulates the proliferation of CD4+ T lymphocytes (108). Same with the Hippo-YAP pathway, Tregs are also characterized by the Yin-Yang dynamics in cancer, as they suppress inflammation-driven tumorigenesis by restraining inflammation, whereas their ability to curb inflammatory cells encourages non-inflammatory tumorigenesis. The "unproductive" immunosuppression even leads to cancer progression (109). Ni et al. (110) reported that YAP was highly expressed in Tregs and enhanced Treg production through the activin-activin receptor signaling axis, causing it to enrich in the tumor. Transcriptional regulation of the star molecule programmed death ligand-1 (PD-L1) gene is another important mechanism for YAP/TAZ to regulate $\mathrm{T}$ cells. PD-L1 is constitutively expressed on the surface of cancer cells, while the receptor of PD-L1, programmed death protein-1 (PD-1), is mostly expressed on the surface of $\mathrm{T}$ cells. Therefore, the interaction between them prevents cancer cells from immune surveillance of $T$ cells (111). YAP/TAZ and PD-L1 have a bidirectional interaction in suppressing anti-tumor $\mathrm{T}$ cell responses. Not only does YAP/TAZ directly upregulate the expression of PD-L1 $(97,112)$, but the overexpression of PD-L1 also effectively increases the level and transcriptional activity of YAP (113).

The first paradox is the relationship between MST1 and Tregs. As an upstream inhibitory molecule of YAP/ TAZ, MST1 is supposed to perform the opposite function to YAP; but it turns out that MST1 can yet enhance Treg function (114) and its deficiency results in autoimmune diseases due to impaired Tregs (115).

The second paradox, also the most concerned, is the finding in an article (116) published in Cell. The authors first observed that the loss of LATS1/2 significantly inhibited tumor growth in a variety of cancer types in mice, e.g., melanoma, breast cancer, squamous cell carcinoma. LATS1/2 are a pivotal factor with growth inhibitory/ tumor-suppressive functions in the Hippo signaling, but without LATS1/2, tumor cells secret nucleic acid-rich EVs to promote TLRs-Myd88/TRIF signaling and type I IFN production, thereby accelerating DC maturation, CD8+ T lymphocyte expansion, and tumor growth arrest (116). Thus, LATS1/2 depletion stimulates the activity of innate and adaptive immunity to completely destroy the TME, resulting in strong anti-tumor immunity. Besides, the researchers added that under physiological conditions, the inhibition of LATS and activation of YAP during wound healing and tissue regeneration both promoted cell proliferation and prevented overgrowth and metastasis from an immunosurveillance perspective. This view complements the above account of the relationship between the Hippo pathway and cell proliferation. Nevertheless, there are limitations to the article. The first is that it acknowledged that the relationship between the Hippo pathway and immune cells in mouse models could not be reproduced in human cancer for the time being (116). Similarly, regarding the relationship between YAP/TAZ and PD-L1 in human cancers, Janse van Rensburg et al. (117) also found that the phenomenon could not be identified in mouse cell lines. Taha et al. (97) made a conjecture that there were species-specific differences in the transcriptional targets of the above molecules. Comparing the Hippo-YAP pathway across species might be a good direction to solve the problem. The second is, other articles $(105,118)$ held converse standpoints: knockdown of LATS1/2 recruited type II macrophages and established an immunosuppressive microenvironment. The difference lies in the properties of the animal model of different cancers. In addition to species specificity, there is also tumor specificity in the HippoYAP pathway, which has been mentioned in some of the contradictory points above.

\section{Conclusions and future prospect}

In this investigation, the aim was to assess recent advance of the Hippo-YAP pathway in oncology and analyze the contradictions that have emerged over the years in a wide range of literature. Considering that all these contradictions have a solid experimental basis, the Hippo pathway should not be simply categorized as a tumor suppressive pathway (Yin) or a tumor promoting pathway (Yang), but should be fully investigated from the view of the Yin-Yang dynamics. More recently, a series of controversial studies $(119,120)$ on breast cancer and the Hippo pathway have been published in Nature. Ma et al. (119) found that LATS1/2 promoted the growth of $\mathrm{ER} \alpha+$ breast cancer cells by maintaining ER $\alpha$ expression, while over-activation of YAP/YAZ was sufficient to inhibit ER $\alpha$ expression, challenging the findings of Britschgi et al. (121) in 2017. Britschgi et al. replied to their query, suggesting that the differences could stem from different subclones, experimental methods, culture conditions, etc. They also acknowledged that LATS1/2 should not be recognized simply as a tumor suppressor. Although the pathogenesis of breast cancer was well established and the literature was published in topical journals, four years later the researchers (119) still 
found a surprising and paradoxical role in breast cancer for one of the Hippo pathway molecules. Thus, we can infer that with many new theories about cancer being proposed, more mechanisms and contradictions of Hippo pathway regulation of cancer will keep coming out in the future. This brings us opportunities for curing cancer and challenges in practical applications at the same time. Many reviews have mentioned that it is yet unknown how to curb YAP/TAZ activation safely and effectively in human patients, and that YAP/TAZ inhibition may have unintended consequences $(89,122)$. However, there is a silver lining: if a therapeutic window for YAP/TAZ activators can be explored in the future, and if YAP/TAZ activators are moderately applied, it is very likely that both the oncogenic potential of YAP/TAZ will be eliminated and the potential of organ regeneration will be fulfilled.

\section{Acknowledgments}

Funding: This work was supported by the National Natural Science Foundation of China (Nos. 81872196 and 81672690).

\section{Footnote}

Reporting Checklist: The authors have completed the Narrative Review reporting checklist. Available at https:// tcr.amegroups.com/article/view/10.21037/tcr-21-1843/rc

Peer Review File: Available at https://tcr.amegroups.com/ article/view/10.21037/tcr-21-1843/prf

Conflicts of Interest: Both authors have completed the ICMJE uniform disclosure form (available at https://tcr.amegroups. com/article/view/10.21037/tcr-21-1843/coif). The authors have no conflicts of interest to declare.

Ethical Statement: The authors are accountable for all aspects of the work in ensuring that questions related to the accuracy or integrity of any part of the work are appropriately investigated and resolved.

Open Access Statement: This is an Open Access article distributed in accordance with the Creative Commons Attribution-NonCommercial-NoDerivs 4.0 International License (CC BY-NC-ND 4.0), which permits the noncommercial replication and distribution of the article with the strict proviso that no changes or edits are made and the original work is properly cited (including links to both the formal publication through the relevant DOI and the license). See: https://creativecommons.org/licenses/by-nc-nd/4.0/.

\section{References}

1. Snigdha K, Gangwani KS, Lapalikar GV, et al. Hippo Signaling in Cancer: Lessons From Drosophila Models. Front Cell Dev Biol 2019;7:85.

2. Xu T, Wang W, Zhang S, et al. Identifying tumor suppressors in genetic mosaics: the Drosophila lats gene encodes a putative protein kinase. Development 1995; 121:1053-63.

3. Fu V, Plouffe SW, Guan KL. The Hippo pathway in organ development, homeostasis, and regeneration. Curr Opin Cell Biol 2017;49:99-107.

4. Mohajan S, Jaiswal PK, Vatanmakarian M, et al. Hippo pathway: Regulation, deregulation and potential therapeutic targets in cancer. Cancer Lett 2021;507:112-23.

5. Harvey KF, Zhang X, Thomas DM. The Hippo pathway and human cancer. Nat Rev Cancer 2013;13:246-57.

6. Rausch V, Hansen CG. The Hippo Pathway, YAP/TAZ, and the Plasma Membrane. Trends Cell Biol 2020;30:32-48.

7. Hong AW, Meng Z, Plouffe SW, et al. Critical roles of phosphoinositides and NF2 in Hippo pathway regulation. Genes Dev 2020;34:511-25.

8. Badouel C, McNeill H. SnapShot: The hippo signaling pathway. Cell 2011;145:484-484.e1.

9. Dong J, Feldmann G, Huang J, et al. Elucidation of a universal size-control mechanism in Drosophila and mammals. Cell 2007;130:1120-33.

10. Ma S, Meng Z, Chen R, et al. The Hippo Pathway: Biology and Pathophysiology. Annu Rev Biochem 2019;88:577-604.

11. Zhao B, Ye X, Yu J, et al. TEAD mediates YAPdependent gene induction and growth control. Genes Dev 2008;22:1962-71.

12. Weiler SME, Lutz T, Bissinger M, et al. TAZ target gene ITGAV regulates invasion and feeds back positively on YAP and TAZ in liver cancer cells. Cancer Lett 2020;473:164-75.

13. He C, Mao D, Hua G, et al. The Hippo/YAP pathway interacts with EGFR signaling and HPV oncoproteins to regulate cervical cancer progression. EMBO Mol Med 2015;7:1426-49.

14. Zhang J, Ji JY, Yu M, et al. YAP-dependent induction of amphiregulin identifies a non-cell-autonomous component of the Hippo pathway. Nat Cell Biol 2009;11:1444-50. 
15. Zhang W, Gao Y, Li P, et al. VGLL4 functions as a new tumor suppressor in lung cancer by negatively regulating the YAP-TEAD transcriptional complex. Cell Res 2014;24:331-43.

16. Dolma S, Lessnick SL, Hahn WC, et al. Identification of genotype-selective antitumor agents using synthetic lethal chemical screening in engineered human tumor cells. Cancer Cell 2003;3:285-96.

17. Dixon SJ, Lemberg KM, Lamprecht MR, et al. Ferroptosis: an iron-dependent form of nonapoptotic cell death. Cell 2012;149:1060-72.

18. Yang WS, SriRamaratnam R, Welsch ME, et al. Regulation of ferroptotic cancer cell death by GPX4. Cell 2014;156:317-31.

19. Brigelius-Flohé R, Maiorino M. Glutathione peroxidases. Biochim Biophys Acta 2013;1830:3289-303.

20. Yang WS, Stockwell BR. Ferroptosis: Death by Lipid Peroxidation. Trends Cell Biol 2016;26:165-76.

21. Friedmann Angeli JP, Schneider M, Proneth B, et al. Inactivation of the ferroptosis regulator $\mathrm{Gpx} 4$ triggers acute renal failure in mice. Nat Cell Biol 2014;16:1180-91.

22. Bridges RJ, Natale NR, Patel SA. System $x^{-}$cystine/ glutamate antiporter: an update on molecular pharmacology and roles within the CNS. Br J Pharmacol 2012;165:20-34.

23. Miess H, Dankworth B, Gouw AM, et al. The glutathione redox system is essential to prevent ferroptosis caused by impaired lipid metabolism in clear cell renal cell carcinoma. Oncogene 2018;37:5435-50.

24. Gao M, Monian P, Quadri N, et al. Glutaminolysis and Transferrin Regulate Ferroptosis. Mol Cell 2015;59:298-308.

25. $\mathrm{Wu}$ J, Minikes AM, Gao M, et al. Intercellular interaction dictates cancer cell ferroptosis via NF2-YAP signalling. Nature 2019;572:402-6.

26. Li J, Cao F, Yin HL, et al. Ferroptosis: past, present and future. Cell Death Dis 2020;11:88.

27. Reinfeld BI, Madden MZ, Wolf MM, et al. Cellprogrammed nutrient partitioning in the tumour microenvironment. Nature 2021;593:282-8.

28. Cairns RA, Harris IS, Mak TW. Regulation of cancer cell metabolism. Nat Rev Cancer 2011;11:85-95.

29. Yang WH, Lin CC, Wu J, et al. The Hippo Pathway Effector YAP Promotes Ferroptosis via the E3 Ligase SKP2. Mol Cancer Res 2021;19:1005-14.

30. Zhu G, Murshed A, Li H, et al. O-GlcNAcylation enhances sensitivity to RSL3-induced ferroptosis via the YAP/TFRC pathway in liver cancer. Cell Death Discov
2021;7:83.

31. Yang WH, Huang $Z$, Wu J, et al. A TAZ-ANGPTL4NOX2 Axis Regulates Ferroptotic Cell Death and Chemoresistance in Epithelial Ovarian Cancer. Mol Cancer Res 2020;18:79-90.

32. van Roy F, Berx G. The cell-cell adhesion molecule E-cadherin. Cell Mol Life Sci 2008;65:3756-88.

33. Love DC, Hanover JA. The hexosamine signaling pathway: deciphering the "O-GlcNAc code". Sci STKE 2005;2005:re13.

34. Peng C, Zhu Y, Zhang W, et al. Regulation of the HippoYAP Pathway by Glucose Sensor O-GlcNAcylation. Mol Cell 2017;68:591-604.e5.

35. Zhang X, Qiao Y, Wu Q, et al. The essential role of YAP O-GlcNAcylation in high-glucose-stimulated liver tumorigenesis. Nat Commun 2017;8:15280.

36. Cho YS, Jiang J. Hippo-Independent Regulation of Yki/ Yap/Taz: A Non-canonical View. Front Cell Dev Biol 2021;9:658481.

37. Sanjaya A, Goenawan H, Setiawan I, et al. Elaborating the Physiological Role of YAP as a Glucose Metabolism Regulator:A Systematic Review. Cell Physiol Biochem 2021;55:193-205.

38. Zhang X, Yu K, Ma L, et al. Endogenous glutamate determines ferroptosis sensitivity via ADCY10-dependent YAP suppression in lung adenocarcinoma. Theranostics 2021;11:5650-74.

39. Komatsu M, Waguri S, Ueno T, et al. Impairment of starvation-induced and constitutive autophagy in Atg7deficient mice. J Cell Biol 2005;169:425-34.

40. Li X, Zhou Y, Li Y, et al. Autophagy: A novel mechanism of chemoresistance in cancers. Biomed Pharmacother 2019;119:109415.

41. Klionsky DJ, Baehrecke EH, Brumell JH, et al. A comprehensive glossary of autophagy-related molecules and processes (2nd edition). Autophagy 2011;7:1273-94.

42. Karsli-Uzunbas G, Guo JY, Price S, et al. Autophagy is required for glucose homeostasis and lung tumor maintenance. Cancer Discov 2014;4:914-27.

43. Mizushima N, Klionsky DJ. Protein turnover via autophagy: implications for metabolism. Annu Rev Nutr 2007;27:19-40.

44. Mizushima N. Autophagy: process and function. Genes Dev 2007;21:2861-73.

45. Klionsky DJ, Codogno P. The mechanism and physiological function of macroautophagy. J Innate Immun 2013;5:427-33.

46. Poillet-Perez L, Xie X, Zhan L, et al. Autophagy maintains 
tumour growth through circulating arginine. Nature 2018;563:569-73.

47. Nassour J, Radford R, Correia A, et al. Autophagic cell death restricts chromosomal instability during replicative crisis. Nature 2019;565:659-63.

48. Wen X, Klionsky DJ. At a glance: A history of autophagy and cancer. Semin Cancer Biol 2020;66:3-11.

49. Kimmelman AC, White E. Autophagy and Tumor Metabolism. Cell Metab 2017;25:1037-43.

50. Yang ZJ, Chee CE, Huang S, et al. The role of autophagy in cancer: therapeutic implications. Mol Cancer Ther 2011;10:1533-41.

51. Tang D, Kang R, Livesey KM, et al. Endogenous HMGB1 regulates autophagy. J Cell Biol 2010;190:881-92.

52. Lee YA, Noon LA, Akat KM, et al. Autophagy is a gatekeeper of hepatic differentiation and carcinogenesis by controlling the degradation of Yap. Nat Commun 2018;9:4962.

53. Jin L, Chen Y, Cheng D, et al. YAP inhibits autophagy and promotes progression of colorectal cancer via upregulating Bcl-2 expression. Cell Death Dis 2021;12:457.

54. Pavel M, Park SJ, Frake RA, et al. $\alpha$-Catenin levels determine direction of YAP/TAZ response to autophagy perturbation. Nat Commun 2021;12:1703.

55. Vousden KH, Prives C. Blinded by the Light: The Growing Complexity of p53. Cell 2009;137:413-31.

56. Belyi VA, Ak P, Markert E, et al. The origins and evolution of the p53 family of genes. Cold Spring Harb Perspect Biol 2010;2:a001198.

57. Lane DP. Cancer. p53, guardian of the genome. Nature 1992;358:15-6.

58. Raj N, Bam R. Reciprocal Crosstalk Between YAP1/Hippo Pathway and the p53 Family Proteins: Mechanisms and Outcomes in Cancer. Front Cell Dev Biol 2019;7:159.

59. Beckerman R, Prives C. Transcriptional regulation by p53. Cold Spring Harb Perspect Biol 2010;2:a000935.

60. Olivier M, Hollstein M, Hainaut P. TP53 mutations in human cancers: origins, consequences, and clinical use. Cold Spring Harb Perspect Biol 2010;2:a001008.

61. Aylon Y, Michael D, Shmueli A, et al. A positive feedback loop between the p53 and Lats2 tumor suppressors prevents tetraploidization. Genes Dev 2006;20:2687-700.

62. Aylon Y, Ofir-Rosenfeld Y, Yabuta N, et al. The Lats2 tumor suppressor augments p53-mediated apoptosis by promoting the nuclear proapoptotic function of ASPP1. Genes Dev 2010;24:2420-9.

63. Vigneron AM, Ludwig RL, Vousden KH. Cytoplasmic ASPP1 inhibits apoptosis through the control of YAP.
Genes Dev 2010;24:2430-9.

64. Momand J, Zambetti GP, Olson DC, et al. The mdm2 oncogene product forms a complex with the $\mathrm{p} 53$ protein and inhibits $\mathrm{p} 53$-mediated transactivation. Cell 1992;69:1237-45.

65. Furth N, Aylon Y. The LATS1 and LATS2 tumor suppressors: beyond the Hippo pathway. Cell Death Differ 2017;24:1488-501.

66. Saladi SV, Ross K, Karaayvaz M, et al. ACTL6A Is CoAmplified with p63 in Squamous Cell Carcinoma to Drive YAP Activation, Regenerative Proliferation, and Poor Prognosis. Cancer Cell 2017;31:35-49.

67. Chen Y, Peng Y, Fan S, et al. A double dealing tale of p63: an oncogene or a tumor suppressor. Cell Mol Life Sci 2018;75:965-73.

68. Tomlinson V, Gudmundsdottir K, Luong P, et al. JNK phosphorylates Yes-associated protein (YAP) to regulate apoptosis. Cell Death Dis 2010;1:e29.

69. Jost CA, Marin MC, Kaelin WG Jr. p73 is a simian correction of human $\mathrm{p} 53$-related protein that can induce apoptosis. Nature 1997;389:191-4.

70. Zaika A, Irwin M, Sansome C, et al. Oncogenes induce and activate endogenous p 73 protein. J Biol Chem 2001;276:11310-6.

71. Yuan ZM, Shioya H, Ishiko T, et al. p73 is regulated by tyrosine kinase c-Abl in the apoptotic response to DNA damage. Nature 1999;399:814-7.

72. Gong JG, Costanzo A, Yang HQ, et al. The tyrosine kinase c-Abl regulates p73 in apoptotic response to cisplatininduced DNA damage. Nature 1999;399:806-9.

73. Levy D, Adamovich Y, Reuven N, et al. Yap1 phosphorylation by c-Abl is a critical step in selective activation of proapoptotic genes in response to DNA damage. Mol Cell 2008;29:350-61.

74. Reuven N, Adler J, Meltser V, et al. The Hippo pathway kinase Lats2 prevents DNA damage-induced apoptosis through inhibition of the tyrosine kinase c-Abl. Cell Death Differ 2013;20:1330-40.

75. Strano S, Monti O, Pediconi N, et al. The transcriptional coactivator Yes-associated protein drives p73 genetarget specificity in response to DNA Damage. Mol Cell 2005; 18:447-59.

76. Basu S, Totty NF, Irwin MS, et al. Akt phosphorylates the Yes-associated protein, YAP, to induce interaction with 143-3 and attenuation of p73-mediated apoptosis. Mol Cell 2003;11:11-23.

77. Strano S, Munarriz E, Rossi M, et al. Physical interaction with Yes-associated protein enhances p73 transcriptional 
activity. J Biol Chem 2001;276:15164-73.

78. Cottini F, Hideshima T, Xu C, et al. Rescue of Hippo coactivator YAP1 triggers DNA damage-induced apoptosis in hematological cancers. Nat Med 2014;20:599-606.

79. Levy D, Adamovich Y, Reuven N, et al. The Yes-associated protein 1 stabilizes $\mathrm{p} 73$ by preventing Itch-mediated ubiquitination of p73. Cell Death Differ 2007;14:743-51.

80. Matallanas D, Romano D, Yee K, et al. RASSF1A elicits apoptosis through an MST2 pathway directing proapoptotic transcription by the p73 tumor suppressor protein. Mol Cell 2007;27:962-75.

81. O'Neill E, Rushworth L, Baccarini M, et al. Role of the kinase MST2 in suppression of apoptosis by the protooncogene product Raf-1. Science 2004;306:2267-70.

82. Sirvent A, Benistant C, Roche S. Cytoplasmic signalling by the c-Abl tyrosine kinase in normal and cancer cells. Biol Cell 2008;100:617-31.

83. Colicelli J. ABL tyrosine kinases: evolution of function, regulation, and specificity. Sci Signal 2010;3:re6.

84. Sawyers CL. Chronic myeloid leukemia. N Engl J Med 1999;340:1330-40.

85. Bai N, Zhang C, Liang $\mathrm{N}$, et al. Yes-associated protein (YAP) increases chemosensitivity of hepatocellular carcinoma cells by modulation of $\mathrm{p} 53$. Cancer Biol Ther 2013;14:511-20.

86. Johnson R, Halder G. The two faces of Hippo: targeting the Hippo pathway for regenerative medicine and cancer treatment. Nat Rev Drug Discov 2014;13:63-79.

87. Silvis MR, Kreger BT, Lien WH, et al. $\alpha$-catenin is a tumor suppressor that controls cell accumulation by regulating the localization and activity of the transcriptional coactivator Yap1. Sci Signal 2011;4:ra33.

88. Moya IM, Halder G. Hippo-YAP/TAZ signalling in organ regeneration and regenerative medicine. Nat Rev Mol Cell Biol 2019;20:211-26.

89. Johnson R, Halder G. The two faces of Hippo: targeting the Hippo pathway for regenerative medicine and cancer treatment. Nat Rev Drug Discov 2014;13:63-79.

90. Stein C, Bardet AF, Roma G, et al. YAP1 Exerts Its Transcriptional Control via TEAD-Mediated Activation of Enhancers. PLoS Genet 2015;11:e1005465.

91. Koo JH, Plouffe SW, Meng Z, et al. Induction of AP-1 by YAP/TAZ contributes to cell proliferation and organ growth. Genes Dev 2020;34:72-86.

92. Mizuno T, Murakami H, Fujii M, et al. YAP induces malignant mesothelioma cell proliferation by upregulating transcription of cell cycle-promoting genes. Oncogene 2012;31:5117-22.
93. Hoxha S, Shepard A, Troutman S, et al. YAP-Mediated Recruitment of YY1 and EZH2 Represses Transcription of Key Cell-Cycle Regulators. Cancer Res 2020;80:2512-22.

94. Huang J, Wu S, Barrera J, et al. The Hippo signaling pathway coordinately regulates cell proliferation and apoptosis by inactivating Yorkie, the Drosophila Homolog of YAP. Cell 2005;122:421-34.

95. He L, Yuan L, Yu W, et al. A Regulation Loop between YAP and NR4A1 Balances Cell Proliferation and Apoptosis. Cell Rep 2020;33:108284.

96. Demaria O, Cornen S, Daëron M, et al. Harnessing innate immunity in cancer therapy. Nature 2019;574:45-56.

97. Taha Z, Janse van Rensburg HJ, Yang X. The Hippo Pathway: Immunity and Cancer. Cancers (Basel) 2018;10:94.

98. Janse van Rensburg HJ, Azad T, Ling M, et al. The Hippo Pathway Component TAZ Promotes Immune Evasion in Human Cancer through PD-L1. Cancer Res 2018;78:1457-70.

99. Zhang Y, Zhang H, Zhao B. Hippo Signaling in the Immune System. Trends Biochem Sci 2018;43:77-80.

100. Valencia-Sama I, Zhao Y, Lai D, et al. Hippo Component TAZ Functions as a Co-repressor and Negatively Regulates $\triangle N p 63$ Transcription through TEA Domain (TEAD) Transcription Factor. J Biol Chem 2015;290:16906-17.

101. Wang G, Lu X, Dey P, et al. Targeting YAP-Dependent MDSC Infiltration Impairs Tumor Progression. Cancer Discov 2016;6:80-95.

102.Pan Z, Tian Y, Cao C, et al. The Emerging Role of YAP/TAZ in Tumor Immunity. Mol Cancer Res 2019;17:1777-86.

103. Urban-Wojciuk Z, Khan MM, Oyler BL, et al. The Role of TLRs in Anti-cancer Immunity and Tumor Rejection. Front Immunol 2019;10:2388.

104. Hagenbeek TJ, Webster JD, Kljavin NM, et al. The Hippo pathway effector TAZ induces TEAD-dependent liver inflammation and tumors. Sci Signal 2018;11:aaj1757.

105. Guo X, Zhao Y, Yan H, et al. Single tumor-initiating cells evade immune clearance by recruiting type II macrophages. Genes Dev 2017;31:247-59.

106. Mou F, Praskova M, Xia F, et al. The Mst1 and Mst2 kinases control activation of rho family GTPases and thymic egress of mature thymocytes. J Exp Med 2012;209:741-59.

107. Fan Y, Gao Y, Rao J, et al. YAP-1 Promotes Tregs Differentiation in Hepatocellular Carcinoma by Enhancing TGFBR2 Transcription. Cell Physiol Biochem 2017;41:1189-98. 
108. Meng K, Butte MJ. Exploring the role of YAP signaling in CD4+T cell biology. J Immunol 2016;196.

109. Wolf D, Sopper S, Pircher A, et al. Treg(s) in Cancer: Friends or Foe? J Cell Physiol 2015;230:2598-605.

110.Ni X, Tao J, Barbi J, et al. YAP Is Essential for TregMediated Suppression of Antitumor Immunity. Cancer Discov 2018;8:1026-43.

111. Wu Y, Zhang C, Liu X, et al. ARIH1 signaling promotes anti-tumor immunity by targeting PD-L1 for proteasomal degradation. Nat Commun 2021;12:2346.

112.Zhou Y, Huang T, Zhang J, et al. Emerging roles of Hippo signaling in inflammation and YAP-driven tumor immunity. Cancer Lett 2018;426:73-9.

113. Tung JN, Lin PL, Wang YC, et al. PD-L1 confers resistance to EGFR mutation-independent tyrosine kinase inhibitors in non-small cell lung cancer via upregulation of YAP1 expression. Oncotarget 2018;9:4637-46.

114.Li J, Du X, Shi H, et al. Mammalian Sterile 20-like Kinase 1 (Mst1) Enhances the Stability of Forkhead Box P3 (Foxp3) and the Function of Regulatory T Cells by Modulating Foxp3 Acetylation. J Biol Chem 2015;290:30762-70.

115. Du X, Shi H, Li J, et al. Mst1/Mst2 regulate development

Cite this article as: Wang R, Zhu G. A narrative review for the Hippo-YAP pathway in cancer survival and immunity: the Yin-Yang dynamics. Transl Cancer Res 2022;11(1):262-275. doi: $10.21037 /$ tcr-21-1843 and function of regulatory $T$ cells through modulation of Foxo1/Foxo3 stability in autoimmune disease. J Immunol 2014;192:1525-35.

116. Moroishi T, Hayashi T, Pan WW, et al. The Hippo Pathway Kinases LATS1/2 Suppress Cancer Immunity. Cell 2016;167:1525-1539.e17.

117.Janse van Rensburg HJ, Yang X. The Hippo pathway and cancer immunity: friend or foe? Oncoscience 2018;5:49-50.

118. Kim W, Khan SK, Liu Y, et al. Hepatic Hippo signaling inhibits protumoural microenvironment to suppress hepatocellular carcinoma. Gut 2018;67:1692-703.

119.Ma S, Wu Z, Yang F, et al. Hippo signalling maintains ER expression and ER+ breast cancer growth. Nature 2021;591:E1-E10.

120. Britschgi A, Couto JP, Bentires-Alj M. Reply to: Hippo signalling maintains ER expression and ER+ breast cancer growth. Nature 2021;591:E11-2.

121. Britschgi A, Duss S, Kim S, et al. The Hippo kinases LATS1 and 2 control human breast cell fate via crosstalk with ER $\alpha$. Nature 2017;541:541-5.

122.Zeng R, Dong J. The Hippo Signaling Pathway in Drug Resistance in Cancer. Cancers (Basel) 2021;13:318. 